\title{
Different Behaviour of Plasma Antioxidant Status after Red Wine Consumption in Subjects of the Same Sex: A Preliminary Report
}

\author{
Marco Giammanco ${ }^{1}$, Danila Di Majo ${ }^{1}$, Gaetano Leto ${ }^{1}$, Carla Flandina ${ }^{1} \&$ Maurizio La Guardia ${ }^{1}$ \\ ${ }^{1}$ Unit of Physiology and Pharmacology, Department of Sport Science and Biomedicine (DISMOT), University \\ of Palermo, Palermo, Italy
}

Correspondence: Marco Giammanco, Department DISMOT, University of Palermo, Via A. Elia, 3, Palermo 90127, Italy. Tel: 39-091-623-6403. E-mail: marco.giammanco@unipa.it

Danila Di Majo, Department DISMOT, University of Palermo, Via A. Elia, 3, Palermo 90127, Italy. Tel: 39-091-623-6405. E-mail: danila.dimajo@unipa.it

Received: June 21, 2012 Accepted: July 4, 2012 Online Published: July 27, 2012

doi:10.5539/jfr.v1n3p302 URL: http://dx.doi.org/10.5539/jfr.v1n3p302

\begin{abstract}
Several studies report that the plasma antioxidant capacity (PAC) increases after a single ingestion of red wine. However, data on the different behaviour of PAC after food intake in subjects of the same sex are still lacking. On the basis of these observations some investigations were undertaken in order to evaluate the effects of red wine intake on PAC in a homogeneous groups of healthy female volunteers $(n=18)$. Additionally, the possible correlation between increase of PAC values and increase in uric acid levels was also assessed. PAC and uric acid concentrations were determined before wine intake and 50,120 and 240 minutes thereafter. The results obtained following these studied highlighted two different patterns of variation of plasma AC values after red wine intake in women. Group "A" exhibits a significant increase in PAC at 120 minutes after wine consumption while group "B" showed a peak level of AC 50 minutes after wine intake. However, no significant correlation was highlighted between increased levels of uric acid and PAC. These results provide a strong argument for the hypothesis that sampling procedures may be one of the confounding factor in studies on the plasma antioxidant status after food or beverage consumption. These preliminary observations indicate that sex-based selection of volunteers should be considered in further investigations.
\end{abstract}

Keywords: antioxidant capacity, red wine, uric acid, crocin bleaching assay, women

\section{Abbreviations}

PAC: plasma antioxidant capacity

TAC: total antioxidant capacity

CBA: crocin bleaching assay

TRAP: total radical trapping antioxidant potential

hSHBG: human sex hormone binding globulin

HDL: high density lipoprotein

LDL: low density lipoprotein

AAPH: 2,20-azobis-(2-amidinopropano-dihydrochloride)

EDTA: ethylene diamine tetraacetic acid

DHBSA: 3,5-dichlor-2-hydroxy-benzolsulfonic acid

GAE: gallic acid equivalent

\section{Introduction}

Experimental, clinical and epidemiologic observations report that the consumption of flavonoid-rich food is associated with a lower incidence of heart disease, ischemic stroke, cancer and other chronic diseases (Joshipura, et al., 1999; Riboli \& Norat, 2003; Bosetti et al., 2005). In these recent years, red wine has been regarded as a "health food". Red wine is known to be a source of polyphenolic compounds with antioxidant properties 
(Rice-Evans, Miller, \& Paganga, 1996). Plasma antioxidant capacity (PAC) is a good parameter of the antioxidant status (Ghiselli et al., 2000). The "antioxidant hypothesis" postulated by Gey (1986), links the high content of antioxidant molecules present in plant food with their health benefits through a direct effect on the oxidative stress (Serafini, 2006). Total antioxidant capacity (TAC) represents the non-enzymatic antioxidants network. TAC comprises the cumulative effects of the whole antioxidants molecules present in plasma matrix. This method provide an integrate parameter rather than the simple sum of measurable antioxidants (Serafini, 2006). Several studies have investigated the effects of wine consumption PAC. The results of these studies highlight a significant increase of PAC value after a single ingestion of red wine (Day \& Stansbie 1995; Serafini, Maiani, \& Ferro-Luzzi, 1998; Duthie et al., 1998; Whitehead, et al., 1995; Maxwell \& Thorpe, 2000). Nonetheless, these studies show that the maximum peak of PAC after red wine consumption can be reached at different times. The differences in results between the studies can be attributed to differences in study design. In fact, investigations directed to assess the pattern of variation of PAC after food or beverages intake in subjects of same sex are scanty. In this context Modun et al. (2008) assessed the effects of red wine consumption on PAC levels in nine healthy males volunteers. These studies showed that the highest peak of PAC mean values is obtained between 60 and 120 minutes. In addition of this, our team has recently observed that in women there is a correlation between the different trend of antioxidant capacity after red wine intake and the different levels of ovarian sex hormones. Therefore, unlike as previously reported in other studies, our observations were focused only on a homogeneous groups of healthy female volunteers. We assessed the short-term effects of red wine intake on PAC by the crocin bleaching assay (CBA). In addition, the possible correlation between increase in PAC levels and increase in plasma urate concentrations after wine consumption was evaluated. We decided to use CBA assay because the informations provided by this method are similar to those provided by the total radical trapping antioxidant potential (TRAP). However, the kinetic approach is more accurate as it takes into consideration both the concentration of antioxidants and their rate constants for the reaction with peroxyl radicals which is overlooked in the TRAP test.

\section{Methods and Materials}

\subsection{Study Design}

Eighteen healthy female volunteers, aged 24-32 years were recruited among the laboratory staff. These subjects were non-smokers and no habitual alcohol consumers. All these subjects were normolipidemic and with a regular ovulatory cycle. None of these volunteers had previous cardiovascular, hepatic, gastrointestinal or renal diseases. Clinical laboratory tests (glucose, cholesterol, triglycerides, HDL, LDL) were within the physiological range. The nutritional status of each subject was evaluated by the bioimpedenziometric analysis and anthropometric measurements (weight, height). The average body mass index (BMI) was $22.97 \pm 2.03 \mathrm{~kg} / \mathrm{m}^{2}$ (range 20.94-25.00 $\mathrm{kg} / \mathrm{m}^{2}$ ). Subjects were instructed to follow a normal caloric diet (1600-1800 Kcal/day) three day before the study, and to avoid consumption the following foods: wine and alcoholic drinks, fruits, vegetables, tea, coffee and chocolate as they can interfere in the evaluation of the PAC. Furthermore, they did not take any vitamin or mineral supplements or drugs which may affect intestinal absorption or the P450 enzymatic system for 3 weeks before the beginning of the study. After overnight fasting, the subjects were asked to consume $300 \mathrm{~mL}$ of red wine. All volunteers drank the same kind of red wine namely Merlot 2004 belonging to the same lot production. The study was carried out in accordance with the declaration of Helsinki and approved by the Local Ethics Committee of the University of Palermo.

\subsection{Chemical and Nutraceutical Characteristics of Red Wine Consumption}

Red wine was chosen between a different set of wines formerly analyzed (Di Majo et al., 2008). The specific wine was selected according to its highest antioxidant capacity $(\mathrm{Ka} / \mathrm{Kc}=17.45 \pm 6.51)$ observed in vitro and total phenolic content $(4.68 \pm 0.43 \mathrm{~g} / \mathrm{L}$ GAE). The values of antioxidant capacity and total phenolic compounds were reported as mean \pm standard deviation values (SD) of five replicate determinations. TAC was measured by CBA and results are expressed as $\mathrm{Ka} / \mathrm{Kc}$. This parameter represents the ratio between reactions rate constants and it describes the antioxidant capacity of red wine to interact with the peroxyl radicals. Total phenolic compounds were evaluated by the Folin-Ciocalteau's method (Di Majo et al., 2008). Results were expressed as g/L of gallic acid equivalents (GAE). The alcoholic content of the red wine consumption was of $32.00 \mathrm{~g} / 300 \mathrm{ml}$ and attributed to each subject a caloric intake of $223.97 \mathrm{Kcal}$ for $300 \mathrm{~mL}$ of red wine.

\subsection{Treatment of Blood Samples}

Venous blood samples were withdrawn by antecubital venipuncture and were collected into EDTA vacutainers (Greiner Bio-one, Austria). Blood samples were obtained before the wine intake (baseline value) and then 50, 120, 240 minutes after wine consumption respectively. Blood samples were immediately centrifuged at 2000 
rpm for $10 \mathrm{~min}$, at $23^{\circ} \mathrm{C}$ avoiding unnecessary exposure to light. The resulting plasma was then aliquoted and stored at $-80^{\circ} \mathrm{C}$ until analysis. The PAC and uric acid levels were determined in each sample by CBA (Tubaro et al., 1998) and by a commercially available kit Fluitest (Biocon Diagnostik Vöhl-Marienhagen) respectively.

\subsection{Analysis of Plasma Antioxidant Capacity by the Crocin Bleaching Assay}

CBA is a competition kinetics assay method for measuring the antioxidant capacity of individual compounds, plant extracts, or plasma (Di Majo et al., 2005; Ordoudi \& Tsimidou, 2006). This protocol was described by Bors (1984), and adapted by Tubaro (1998). The advantage of this method consists in the possibility to be used in both lipophilic and hydrophilic environment (Finotti \& Di Majo, 2003). Another advantage over the others in that it is able to detect either the antioxidant or the pro-oxidant activity of the compound or mixture of compounds under analysis. This method is based on the crocin bleaching as result of its oxidation induced by peroxyl radicals produced from 2,20-azobis-(2-amidinopropano-dihydrochloride) (AAPH Wako Chemicals). Crocin was extracted twice from authentic commercial saffron (origin grade) as described by Ordoudi and Tsimidou (2006). The estimation of its concentration to $\sim 3 \mu \mathrm{M}$ was based on an extinction coefficient as previously described by Finotti and Di Majo (2003), $\varepsilon^{\mathrm{MeOH}}=1.33 * 10^{5} \mathrm{~mol}^{-1} \mathrm{~cm}^{-1}$. Crocin working solutions were daily prepared in methanol (Merck). All treatments were performed away from a direct exposure to light. A certain volume of crocin working solution was diluted with phosphate buffer $(66.7 \mathrm{mM}, \mathrm{pH} 7.4)$ to $50 \mathrm{~mL}$ (total volume) so that the $\mathrm{A}_{443}$ value was $\sim 1$. Then AAPH stock solution $(12.5 \mathrm{mM})$ was prepared in distilled water. Plasma was not diluted. The reaction mixture, in presence of plasma samples, contained $0.15 \mathrm{~mL}$ of AAPH, $0.15 \mathrm{~mL}$ of diluted crocin, increasing amount $(0.15 \mathrm{~mL}-0.02 \mathrm{~mL})$ of plasma and distilled water to a final volume of $1 \mathrm{~mL}$. Conversely, in absence of plasma, the mixture contained $0.15 \mathrm{~mL}$ of AAPH, $0.15 \mathrm{~mL}$ of crocin and distilled water to a final volume of $1 \mathrm{~mL}$. The reactions were started by the introduction of source of radicals (AAPH) and were runned at $40^{\circ} \mathrm{C}$. The bleaching rate of crocin becomes linear approximately 2 minutes after the addition of the diazocompound and a correspondent decrease of absorbance at $443 \mathrm{~nm}$ was monitored for 10 minutes, by using a Beckman $640 \mathrm{UV} /$ visible spectrophotometer, against a blank. Loss in absorbance values within $10 \mathrm{~min}$ of reaction both in the absence $(\Delta \mathrm{Vo})$ and in the presence of increasing amounts of plasma sample $(\Delta \mathrm{Va})$ was calculated. Different plasma concentrations $(0.15 \mathrm{~mL}-0.02 \mathrm{~mL})$ were also challenged so that linear regression curves of relative rates $(\Delta \mathrm{Vo} / \Delta \mathrm{Va})$ against [plasma samples]/[crocin] ratios could be generated. The five-point linear regression slopes representing the relative rate costants $\left(\mathrm{K}_{\mathrm{rel}}=\mathrm{K}_{\mathrm{a}} / \mathrm{K}_{\mathrm{c}}\right)$ were calculated according to the following equation (1):

$$
\mathrm{Vo} / \mathrm{Va}=1+\mathrm{K}_{\mathrm{a}} / \mathrm{K}_{\mathrm{c}} *[\mathrm{~A}] /[\mathrm{C}]
$$

where $\mathrm{Ka}$ is the rate constant for the reaction between antioxidant and peroxyl radicals; $\mathrm{Kc}$ is the rate constant for the reaction between crocin and peroxyl radicals; [A] is the concentration of plasma samples and [C] is the concentration of crocin. All the concentrations are expressed as $\% \mathrm{v} / \mathrm{v}$.

The $\mathrm{K}_{\mathrm{a}} / \mathrm{K}_{\mathrm{c}}$ ratio values indicate the relative antioxidant capacity (AC) of plasma samples to interact with peroxyl radicals.

Vo is the rate of the reaction of crocin with peroxyl radical and can be calculated with the following formula:

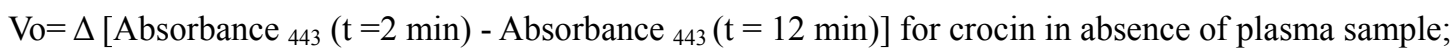

$\mathrm{Va}$ are the rates of the reaction of the crocin with the peroxyl radicals in the presence of different levels of plasma $(0.15 \mathrm{~mL}-0.02 \mathrm{~mL})$ and it was determined according to the following formula:

$\mathrm{Va}=\Delta\left[\right.$ Absorbance $_{443}(\mathrm{t}=2 \mathrm{~min})-$ Absorbance $\left._{443}(\mathrm{t}=12 \mathrm{~min})\right]$ for each concentration $(\% \mathrm{v} / \mathrm{v})$ of plasma sample analyzed.

\subsection{Quantitative Determination of Uric Acid}

An enzymatic in vitro test was used for the quantitative determination of uric acid in human plasma. This method eliminates the interferences related to chemical oxidation. This assay is a slight modification of the colorimetric method. In this reaction the peroxide reacts in the presence of peroxidase, DHBSA (3,5-dichlor-2-hydroxy-benzolsulfonic acid) and 4-aminoantipyrine to form a quinoneimine dye. The intensity of the red colour $(\lambda=555 \mathrm{~nm})$ is proportional to the uric acid concentration in the sample. The uric acid concentration was measured by the follow equation (2):

$$
\Delta \mathrm{A}_{\text {sample/ }} \Delta \mathrm{A}_{\text {calibrator }} * \text { calibrator concentration }
$$

Where calibrator concentration is $6 \mathrm{mg} / \mathrm{dl}(356.9 \mu \mathrm{mol} / \mathrm{l})$ and expressed as $\mathrm{mg} / \mathrm{dl}$ 


\subsection{Statistical Analysis}

Results are reported as mean \pm SD of five determinations. Data were analyzed by the Student's test (t-Student) for paired samples. $P$ values $<0.05$ were considered statistically significant

\section{Results}

The results identified two groups according to the trend of the PAC values observed after red wine intake. Group "A" included ten subjects while group "B" consisted of eight subjects. Table 1 reports the mean values \pm SD related to all the subjects enrolled for the study. The PAC values for each group at time zero and 50 min., zero and 120 min.; 50 and 120min.; zero and 240 min.; 50 and 240min. and 120 and $240 \mathrm{~min}$. were compared. Before red wine intake (baseline values) group A showed antioxidant capacity values comparable with those determined in group B $(p>0.05)$. The differences between the two groups were observed after the interaction with the wine matrix (see Table 1).

Table 1. Mean PAC values measured by CBA in human female volunteers before wine intake (baseline) and at 50,120 and $240 \mathrm{~min}$ after consumption of $300 \mathrm{ml}$ of red wine.

\begin{tabular}{lll}
\hline Volunteers & Time (minutes) & Plasma AC $(\mathrm{Ka} / \mathrm{Kc})$ \\
\hline \multirow{2}{*}{ Group A $(\mathrm{n}=10)$} & 0 & $14.67 \pm 4.67$ \\
& 50 & $15.42 \pm 2.03$ \\
& 120 & $24.79 \pm 4.45^{* *}$ \\
Group B $(\mathrm{n}=8)$ & 240 & $16.83 \pm 5.16^{\S}$ \\
& 0 & $13.04 \pm 4.72$ \\
& 50 & $23.16 \pm 4.21^{* * *}$ \\
Groups A+B $(\mathrm{n}=18)$ & 120 & $14.97 \pm 2.80^{*}$ \\
& 240 & $19.18 \pm 1.67$ \\
& 0 & $14.90 \pm 4.50$ \\
& 50 & $18.04 \pm 5.50$ \\
& 120 & $21.40 \pm 5.95$ \\
& 240 & $18.14 \pm 4.81$
\end{tabular}

Data are expressed as means \pm SD of 5 duplicate determonations. In parenthesis the number of subject of group $\mathrm{A}$, group $\mathrm{B}$, and total subjects $(\mathrm{A}+\mathrm{B}) . \mathrm{Ka} / \mathrm{Kc}$ value is the ratio between the constants of kineticts reaction and describes the antioxidant capacity of plasma to interact with the peroxyl radicals. Statistical significance between group "A" and group "B" at different times were determined by the Student t-test for paired samples.

** $p<0.01$ (baseline vs 120 min after wine intake);

$\S_{p}<0.01$ (240 vs $120 \mathrm{~min}$ after wine intake);

${ }^{*} p<0.05$ (120 i vs 50 min-after wine intake);

${ }^{* * *} \mathrm{p}<0.01$ (baseline vs $50 \mathrm{~min}$. after wine intake)

\subsection{Different Trends of Plasma Antioxidant Capacity in the Two Groups of Women}

Average increases in PAC in the two group of women are reported in Figure 1. 
Group A of women $\square$ Group B of women

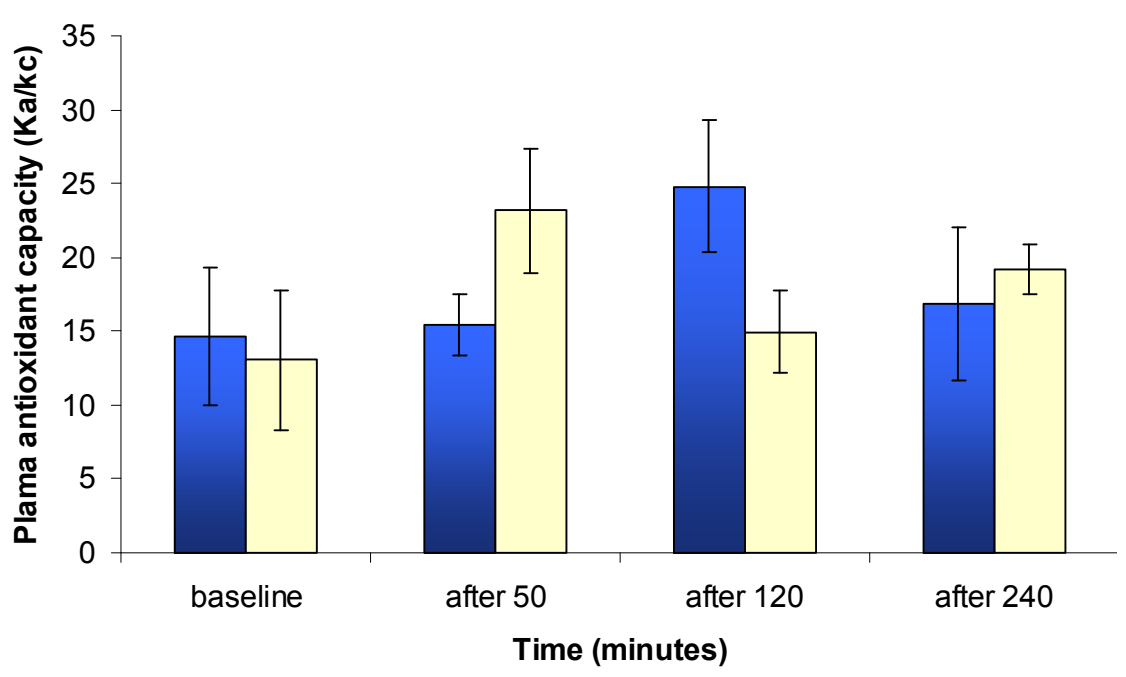

Figure 1. PAC before (baseline) and 50, 120, 240 min after red wine consumption evaluated by CBA in Group A $(\mathrm{n}=10)$ and Group $\mathrm{B}(\mathrm{n}=8)$. Data are the mean $\pm \mathrm{SD}$ of 5 duplicate determinations . PAC value is expressed as $\mathrm{Ka} / \mathrm{Kc}$ and indicates the relative antioxidant capacity (AC) of plasma samples to interact with peroxyl radicals

The statistic analysis of the data show that, in Group A, PAC levels, determined before wine intake were significantly different $(\mathrm{p}<0.01)$ from those measured 120 min-after wine ingestion. The rate of increase was $69 \%$ after $120 \mathrm{~min}$ from red wine intake. However, in this group no significant difference $(p>0.05)$ was observed in the value determined after 50 minutes as compared to baseline value. PAC remained significantly elevated after 240 minutes of red wine intake. On the other hand, in group B the highest values were observed after 50 minutes from red wine intake $(p<0.01)$. PAC levels increased of $77.6 \%$ at $50 \mathrm{~min}$ as compared to the baseline value. These levels significantly decreased 120 min after wine consumption $(p=0.01)$ and then returned to baseline values after $240 \mathrm{~min}$.

\subsection{Analysis of Uric Acid Levels after Red Wine Intake}

Several authors report a significant correlation between an increase in total plasma antioxidant capacity and the increase in plasma urate concentrations after wine consumption (Day \& Stansbie 1995; Lotito \& Frei, 2006). Our studies showed the same trend of uric acid concentrations in both groups A and B of women (Table 3). The highest peak value in all these subjects was reached 50 minutes after red wine consumption. In group A, mean increase in uric acid concentration was $81 \%$ as compared to baseline values $(p<0.05)$. These levels returned back to the baseline value after $120 \mathrm{~min}$. On the contrary, an increase of $33 \%$ in uric acid concentration at $50 \mathrm{~min}$ after red wine consumption was observed in group B. Uric acid levels did not settle back to the baseline value after $240 \min (p<0.01)$. 
Table 3. Mean uric acid plasma concentrations in healthy female volunteers

\begin{tabular}{lll}
\hline Volunteers & Time (minutes) & Uric acid concentrations $(\mathrm{mg} / \mathrm{dl})$ \\
\hline Group A (n=10) & 0 & $4.49 \pm 1.73$ \\
& 50 & $8.13 \pm 1.60^{*}$ \\
& 120 & $6.22 \pm 1.85$ \\
Group B(n=8) & 240 & $4.61 \pm 0.51$ \\
& 0 & $3.21 \pm 0.46$ \\
& 50 & $4.26 \pm 0.44^{* *}$ \\
Groups A+B (n=18) & 120 & $4.26 \pm 0.66$ \\
& 240 & $4.19 \pm 0.44^{\S}$ \\
& 0 & $3.60 \pm 1.10$ \\
& 50 & $5.79 \pm 2.20$ \\
& 120 & $5.13 \pm 1.30$ \\
& 240 & $4.39 \pm 0.45$ \\
\hline
\end{tabular}

Results are reported as means \pm SD of of 5 duplicate determinations. Data were analyzed the Student -t-test for paired samples.

${ }^{*} \mathrm{p}<0.05$ (baseline vs $50 \mathrm{~min}$. after wine intake);

${ }^{* *} \mathrm{p}<0.05$ (baseline vs 50 min. after wine intake);

${ }^{\S} \mathrm{P}<0.01$ (baseline vs 240 min.after wine intake).

\section{Discussion}

\subsection{Analysis of the Plasma Antioxidant Capacity after Red Wine Intake in Women}

The present study shows that the intake of red wine induce a time-dependent increase of the PAC in healthy female subjects. Interestingly, our results highlight that, in these subjects, two main different time -dependent pattern of variations of plasma antioxidant values can be observed following a red wine intake. In fact, in a group of ten subjects (group A) the maximum antioxidant capacity value is reached later than in the other eight subjects (group B). These results are consistent with those reported by Fernández-Pachón (2005) who show that the difference in these results can be due to individual variability in the absorption and metabolism processes. Moreover, Lotito and Frei (2006) indicate PAC may be significantly affected by non-antioxidant constituents which are present in the diet and which may alter the uptake, tissue mobilization or metabolism of endogenous or exogenous antioxidants. These results may be also, in part, explained with the possible interaction of some polyphenols, present in the red wine with the circulating sex-hormone binding globulin (hSHBG). In fact, according to Déchaud et al. (1999) some flavonoids such as naringenin may displace endogenous testosterone and $17-\beta$-estradiol from hSHBG binding sites. This phytoestrogen binds hSHBG by a reversible and competitive binding activity for both testosterone and $17-\beta$-estradiol and with no apparent decrease in the number of hSHBG binding sites. On the basis of these considerations it is conceivable to hypothesize that the different trend of antioxidant capacity after red wine intake we observed may be due to the different levels of circulating sex hormones related to the phase of the ovarian cycle in which the woman was at the time of the study. This hypothesis is supported by some our preliminary observations on a small number of subject which highlight that, after red wine consumptions, the trend of PAC values during the follicular phase is comparable with the trend of values observed in group A, whereas the trend in ovulation and luteal phases overlaps with the group B (unpublished observations). These preliminary observations suggest a possible correlation between variation patter of circulating sex hormones and antioxidant activity level, after food intake. Therefore these studies warrant further investigations with a wide number of subjects and also suggest the need of a sex-based selection of the subjects involved in these studies.

\subsection{Role of Uric Acid on the Different Trend of Plasma Antioxidant Capacity in Two Groups of Women}

The altered levels of uric acid cannot account for the observed increase in PAC after the administration of red wine. In fact, our experiments do not highlight any significant relationship between increased level of uric acid 
and antioxidant capacity. Group A shows a different pattern of both uric acid and antioxidant capacity after red wine intake. The highest peak of antioxidant capacity is reached at $120 \mathrm{~min}$ whereas the highest level of uric acid was observed after $50 \mathrm{~min}$. In the group B the highest peak of antioxidant capacity overlaps with that of uric acid. However the antioxidant capacity settles back to the baseline value after $240 \mathrm{~min}$ while the levels of uric acid remain constant up to $240 \mathrm{~min}$ after red wine intake. This result is consistent with those reported by other authors (Fernández-Pachón et al., 2005) which show that consumption of red wine appears to increase PAC values by two separate mechanisms and that wine phenols and plasma urate may contribute to elicit this effect (Modun et al., 2008).

\section{Conclusion}

The present study show that the intake of red wine induce a time dependent increase of the plasma antioxidant activity in healthy female subjects. These data may contribute to settle the discrepancies in the results obtained from different trials studies (Serafini, Maiani, \& Ferro-Luzzi, 1998; Duthie et al, 1998; Fernández-Pachón et al., 2005; Natella et al., 2001). Moreover, these observations indicate that further investigations with a wide number of subjects are needed to better assess the relationship between trend of sex hormones levels during the three phases of the ovarian cycle and variations of the antioxidant status after the consumption of rich-in-antioxidants foods. This results strongly support the concept that the sampling procedures may be one of the confounding factors in studies on the plasma antioxidant status after food or beverage consumption. We think that sex-based selection of volunteers, and ovarian cycle are essential prerequisite to better design future clinical studies aimed at evaluating the preventive role of flavonoids in age related diseases. Moreover, the present study, performed by using CBA, further confirms previous observations, which show that the altered levels of uric acid cannot account for the increase values of PAC observed after red wine consumption.

\section{References}

Bors, W., Michel, C., \& Saran, M. (1984). Inhibition of the bleaching of the carotenoid crocin. A rapid test for quantifying antioxidant activity. Bioch Biophy Acta, 796, 312-319.

Bosetti, C., Spertini, L., Parpinel, M., Gnagnarella, P., Lagiou, P., Negri, E., ... La Vecchia, C. (2005). Flavonoids and breast cancer risk in Italy. Cancer Epidemiology, Biomarkers \& Prevention, 144, 805-808. http://dx.doi.org/10.1158/1055-9965.EPI-04-0838

Day, A., \& Stansbie D. (1995). Cardioprotective effect of red wine may be mediated by urate. Clinical Chemistry, 41, 1319-1320.

Déchaud, H., Ravard, C., Claustrat, F., Brac de la Perrière, A., \& Pugeat, M. (1999). Xenoestrogen interaction with human sex hormone-binding globulin (hSHBG). Steroids, 64, 328-334. http://dx.doi.org/10.1016/S0039-128X(98)00114-7

Di Majo, D., Giammanco, M., La Guardia, M., Tripoli, E., Giammanco, S., \& Finotti E. (2005). Flavanones in Citrus fruit: Structure-antioxidant activity relationships. Food Research International, 38, 1161-1166. http://dx.doi.org/10.1016/j.foodres.2005.05.001

Di Majo, D., La Guardia, M., Giammanco, S., La Neve, L., \& Giammanco, M. (2008). The antioxidant capacity of red wine in relationship with its polyphenolic costituents. Food Chemistry, 111, 45-49. http://dx.doi.org/10.1016/j.foodchem.2008.03.037

Duthie, G. G., Pedersen, M. W., Gardner, P. T., Morrice, P. C., Jenkinson A. McE., Mc Phail, D. B., \& Steele G. M. (1998). The effect of whisky and wine consumption on total phenol content and antioxidant capacity of plasma from healthy volunteers. European Journal of Clinical Nutrition, 52, 733-736. http://dx.doi.org/10.1038/sj.ejen.1600635

Fernández-Pachón, M. S., Villaño, D., Troncoso, A. M., \& García-Parrila, M. C. (2005). Antioxidant capacity of plasma after red wine intake in human volunteers. Journal of Agricultural and Food Chemistry, 53, 5024-5029. http://dx.doi.org/10.1021/jf0501995

Finotti, E., \& Di Majo, D. (2003). Influence of solvents on the antioxidant property of flavonoids. Nahrung/Food, 47,186-187. http://dx.doi.org/10.1002/food.200390043

Gey, K. F. (1986). On the antioxidant hypothesis with regard to arteriosclerosis. Bibliotheca Nutritio et Dieta, 37, 53-91.

Ghiselli, A., Natella, F., Guidi, A., Montanari, L., Fantozzi, P., \& Scaccini C. (2000). Beer increases plasma antioxidant capacity in human. The Journal of Nutritional Biochemitry, 11, 76-80. http://dx.doi.org/10.1016/S0955-2863(99)00077-7 
Joshipura, K. J., Ascherio, A., Manson, J. A. E., Stampfer, M. J., Rimm, E. B., Speizer, F. E., ... Willett, W. C. (1999). Fruit and vegetable intake in relation to risk of ischemic stroke. JAMA, 282, 1233-1239. http://dx.doi.org/10.1001/jama.282.13.1233

Lotito, S. B., \& Frei, B. (2006). Consumption of flavonoid-rich foods and increased plasma antioxidant capacity in humans: cause, consequence or epiphenomenon? Free Radical Biology \& Medicine, 41, 1727-1746. http://dx.doi.org/10.1016/j.freeradbiomed.2006.04.033

Maxwell, S., \& Thorpe, G. (2000). Impact of red wine on antioxidant status in vivo. European Heart Journal, 21, 1482-1483. http://dx.doi.org/10.1053/euhj.2000.2235

Modun, D., Music, I., Vukovic, J., Brizic, I., Katalinic, V., Obad, A., ... Boban, M. (2008). The increase in human plasma antioxidant capacity after red wine consumption is due to both plasma urate and wine polyphenols. Atherosclerosis, 197, 250-256. http://dx.doi.org/10.1016/j.atherosclerosis.2007.04.002

Natella, F., Ghiselli, A., Guidi, A., Ursini, F., \& Scaccini, C. (2001). Red wine mitigates the postprandial increase of LDL susceptibility to oxidation. Free Radical Biology \& Medicine, 30, 1036-1044. http://dx.doi.org/10.1016/S0891-5849(01)00504-4

Ordoudi, S. A., \& Tsimidou, M. Z. (2006). Crocin Bleaching Assay Step by Step: Observations and Suggestion for an Alternative Validated Protocol. Journal of Agricultural and Food Chemistry, 54, 1663-1661. http://dx.doi.org/10.1021/jf052731u

Riboli, E., \& Norat, T. (2003). Epidemiologic evidence of the protective effect of fruit and vegetables on cancer risk. The American Journal of Clinical Nutrition, 78, 559S-569S.

Rice-Evans, C., Miller, N. J., \& Paganga, G. (1996). Structure-antioxidant activity relationships of flavonoids and phenolic acids. Free Radical Biology \& Medicine, 20, 933-956. http://dx.doi.org/10.1016/0891-5849(95)02227-9

Serafini, M. (2006). The role of antioxidant in disease prevention. Medicine, 34, 533-535. http://dx.doi.org/10.1053/j.mpmed.2006.09.007

Serafini, M., Maiani, G., \& Ferro-Luzzi A. (1998). Alcol-free red wine enhances plasma antioxidant capacity in human. Journal of Nutrition, 128, 1003-1007.

Tubaro, F., Ghiselli, A., Rapuzzi, P., Maiorino, M., \& Urini, F. (1998). Analysis of plasma antioxidant capacity by competition kinetics. Free Radical Biology \& Medicine, 24, 1228-1234. http://dx.doi.org/10.1016/S0891-5849(97)00436-X

Whitehead, T. P., Robinson, D., Allaway, S., Syms J., \& Hale A. (1995). A Effect of red wine ingestion on the antioxidant capacity of serum. Clinical Chemistry, 41, 32-35. 